\title{
Paquimeningite Hipertrófica
}

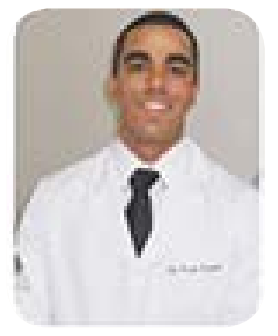

Tiago Santana Santos Queiroz', Matheus Mendes Pires', Itana Naiara Ribeiro ${ }^{1}$, Pedro Antonio Pereira de Jesus ${ }^{1}$

\section{RESUMO}

Paquimeningite Hipertrófica (PMH) é uma condição onde ocorre o envolvimento difuso ou localizado da dura mater em sua porção craniana ou medular, caracterizado por um espessamento da mesma. Pode estar associada a doenças sistêmicas inflamatórias, infecciosas e neoplásicas ou acontecer de forma idiopática (PMHI), quando não se encontra uma causa específica. O diagnóstico é obtido através dos achados clínicos, exames laboratoriais, exames de imagem e estudo histopatológico. O tratamento deve ser individualizado, com base na causa encontrada durante a investigação, geralmente havendo indicação de corticoterapia.

PALAVRAS-CHAVE: paquimeningite hipertrófica; espessamento dural; doença por lgG4.

KEY-WORDS: hypertrophic pachymeningitis; dural thickening; IgG4-related disease.

\section{INTRODUÇÃO}

As meninges consistem em três membranas que envolvem e protegem o sistema nervoso central (SNC). Podendo ser agrupadas como paquimeninge (dura mater) e leptomeninge (aracnoide e pia mater) ${ }^{1}$. A dura mater está presente na interface entre o encéfalo e o crânio, entre a medula e as vértebras, bem como formando os seios durais.

Em 1869 foi descrita por Charcot e Joffry uma "condição na qual ocorre um acometimento das leptomeninges, tornando-as opacas e espessas e subsequentemente aderidas à dura mater e medula."2. Hoje denominamos paquimeningite hipertrófica $(\mathrm{PMH})$, a condição caracterizada pelo espessamento da dura mater (localizado ou difuso), podendo ou não estar associado a um processo inflamatório crônico. Foram descritos na literatura vários casos compatíveis com esse diagnóstico, apresentando envolvimento das meninges tanto do segmento espinhal quanto intracrania- no ${ }^{3}$. A PMH é considerada uma doença rara e existem poucos dados sobre sua prevalência. No Japão, um estudo populacional mostrou que sua prevalência naquela população é de 0.949/100 $000^{11}$. Na forma idiopática da doença, os indivíduos do sexo masculino são afetados discretamente em maior frequência do que os do sexo feminino $(1: 0,75)$ e tipicamente há maior incidência entre a sexta e sétima décadas de vida ${ }^{4,11}$, mas a doença pode acometer indivíduos de qualquer faixa etária, inclusive há casos relatados de $\mathrm{PMH}$ em crianças $^{19}$.

Diversas doenças inflamatórias sistêmicas foram relacionadas com $\mathrm{PMH}$, incluindo neurossarcoidose, granulomatose com poliangeíte (antigamente denominada "granulomatose de Wegner"), síndrome de Sjogren, artrite reumatoide, arterite de células gigantes, síndrome de Behçet e mais recentemente a doença por $\operatorname{lgG} 4^{5,6,13}$. Causas infecciosas também foram descritas, incluindo sífilis, tuberculose e doença de Lyme ${ }^{7,8}$. Há também relatos de envolvimento dural relacionado a processos neoplásicos, mais comumente originados de lesões primárias de mama, próstata, pulmões e células sanguíneas ou à extensão de neoplasias de base de crânio ou meningioma ${ }^{4,9}$. Um importante diagnóstico diferencial é a síndrome de hipotensão intracraniana. A mesma pode se apresentar com sintomas e achados radiológicos semelhantes (pseudoespessamento meníngeo). É fundamental afastar essa condição, pois seu tratamento específico é bem diferente da $\mathrm{PMH}$, sendo baseado em repouso, hidratação, "blood patch" e, em alguns casos, neurocirurgia. Quando não é possível identificar uma causa específica para os achados da $\mathrm{PMH}$, pode ser utilizada a terminologia Paquimeningite Hipertrófica Idiopática (PMHI) ${ }^{4}$.

\section{APRESENTAÇÃO CLÍNICA}

Os sinais e sintomas da PMHI dependem do local de acometimento das meninges. As possibilidades de apresentação clínica são bastante amplas, sendo o 
mais habitual encontrar cefaleia e sintomas relacionados ao envolvimento de nervos cranianos, nos casos de acometimento craniano ${ }^{10}$. A doença pode se apresentar com curso monofásico, progressivo ou em forma de surtos.

Em um estudo populacional japonês, o envolvimeno craniano predominou, acometendo $89,3 \%$ dos indivíduos com $\mathrm{PMH}$ e o medular ocorreu em apenas $13,2 \%$. Os sintomas mais prevalentes na abertura do quadro são: cefaleia $(35,2 \%)$, perda visual $(13,2 \%)$, diplopia $(12,6 \%)$ e sintomas otológicos $(9,4 \%)$. Durante a evolução, cerca de $23,9 \%$ dos pacientes apresentam fraqueza (facial ou apendicular), 28,3\% algum grau de comprometimento sensorial, e outros ainda cursam com sintomas disautonômicos.

De modo geral, observa-se boa correlação entre as queixas apresentadas e os achados do exame físico ${ }^{11}$. No exame físico dos pacientes podemos observar paralisia de um nervo craniano isolado ou de múltiplos nervos cranianos, que é um achado frequente. Além disso podemos verificar fraqueza muscular, reflexos osteotendíneos exacerbados, presença de sinais de irritação meníngea e alteração de sensibilidade. Em alguns casos é possível verificar sinais de disautonomia, bem como déficit cognitivo ${ }^{11,17}$. O exame do fundo de olhos pode demonstrar presença de papiledema, devido à síndrome de hipertensão intracraniana ou presença de papilite, devido à inflamação do nervo óptico.

\section{INVESTIGAÇÃO}

A investigação complementar deve incluir estudo completo do LCR, velocidade de hemossedimentação (VHS), proteína $C$ reativa (PCR), dosagem de enzima conversora de angiotensina (ECA), fator antinuclear (FAN), fator reumatoide (FR), anticorpos citoplasmáticos antineutrófilos ANCA, Anti-dsDNA, Anti-SSA, Anti -SSB, VDRL, PPD, eletroforese de proteínas séricas, dosagem de lgG4 sérica/LCR ${ }^{6,10}$.

É mandatória a realização de exame de imagem, guiado pela suspeita topográfica. O exame de tomografia é de pouca utilidade para o diagnóstico. A ressonância magnética (RM), seja de crânio ou de medula, conforme o sítio de acometimento da doença, é o melhor exame de imagem para diagnóstico de $\mathrm{PMH}$.

No exame de ressonância magnética, o achado mais comum é a presença de espessamento da dura mater com realce na sequência T1 após infusão de gadolíneo $^{12}$ (Figura 1). As lesões vistas à RM podem ter aspecto nodular ou não-nodular, sendo comum o envolvimento da foice cerebral e tenda do cerebelo ${ }^{4}$.
A paquimeningite medular geralmente envolve as regiões cervical e torácica alta $^{13}$.

Frequentemente, os achados da RM podem ser insuficientes para afastar outras condições como linfoma, tuberculose, metástase dural (Figuras 2 e $3)^{8,10,14,15,16}$. Desse modo, habitualmente se faz necessária a realização de biopsia da meninge para estudo anatomopatológico e estabelecimento de um diagnóstico definitivo. O estudo histopatológico pode evidenciar infiltrado inflamatório (predomínio de linfócitos e histiócitos epiteliais), com ou sem formação de granuloma, mas sem evidência de vasculite e com ausência de agentes infecciosos ${ }^{4,10,11}$. Quando associado à doença por IgG4 pode haver a presença de células positivas para IgG4, fibrose em padrão esporiforme e flebite obliterativa $^{17}$ (figura 4).

\section{TRATAMENTO}

A escolha e a resposta ao tratamento estão diretamente relacionadas ao diagnóstico de uma causa específica. Quando identificadas as causas infecciosas, devem ser utilizadas as drogas específicas para cada agente (eg. Treponema pallidum, Mycobacterium tuberculosis, Borrelia burgdorferi). Mediante causas neoplásicas, sejam elas primárias (meningioma, tumores de base de crânio) ou secundárias (linfomatose ou carcinomatose meníngea), deve-se acionar o serviço de neurocirurgia e oncologia para definir melhor proposta terapêutica.

Caso sejam identificadas causas inflamatórias ou feito diagnóstico de PMHI, podem ser utilizados os corticoesteroides, preferencialmente pela via oral. Há relatos na literatura de melhora dos sintomas como cefaleia e até mesmo dos déficits neurológicos focais com uso de prednisona nas doses diárias entre 60-80mg. Como trata-se de uma condição crônica, geralmente há demanda para uso prolongado de corticoides e a resposta nem sempre é satisfatória. $\mathrm{Em}$ casos selecionados, pode haver benefício com a associação de imunossupressores (metotrexate e azatiopina) e eventualmente se faz necessária uma abordagem mais agressiva com metilprednisolona venosa ${ }^{4,10,11}$. Quando associado à doença por $\lg G 4$, os imunossupressores mostraram uma eficácia muito variável, sendo que muitos pacientes permanecem em uso de altas doses de corticoide oral como tratamento de manutenção ${ }^{17}$. Boa resposta ao uso de rituximab tem sido relatada em pacientes com apresentação sistêmica da doença por $\lg \mathrm{G} 4$, mas ainda com baixo nível de evidência nas apresentações com paquimeningite isolada ${ }^{18}$. 


\section{IMAGENS}

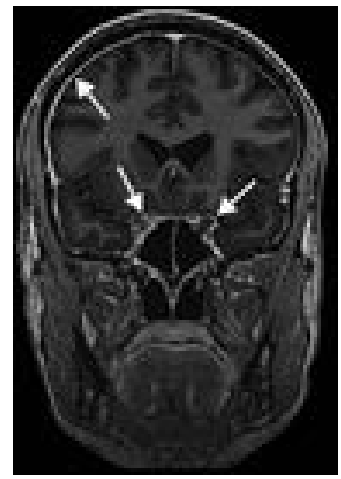

Figura 1 - RM em corte coronal, T1 com infusão de gadolíneo, evidenciando realce dural difuso com envolvimento bilateral dos canais ópticos ${ }^{12}$.

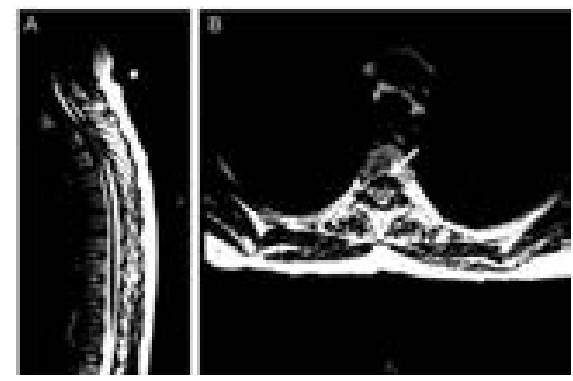

Figura 2 - (A) RM com corte sagital em T2, demonstrando lesão dural hipointensa no interior do canal medular (T2-T5), se extendendo anteriormente. (B) RM com corte axial em T2, demonstrando massa dural (T3-T4) na porção anterior do canal medular, após exclusão de causas secundárias foi feito o diagnóstico de Paquimeningite hipertrófica idiopática ${ }^{16}$.

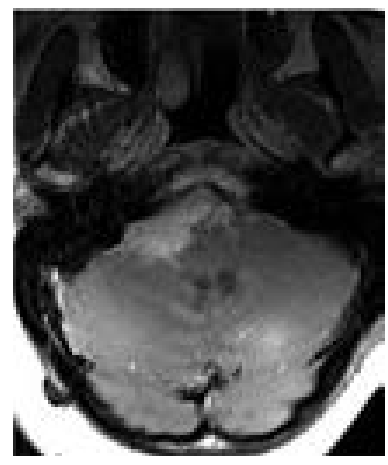

Figura 3 - RM evidenciando lesão dural envolvendo canal cervical rostral e fossa posterior com efeito de massa, exame histopatológico evidenciando inflamação com presença de "granuloma não-caseoso"10.

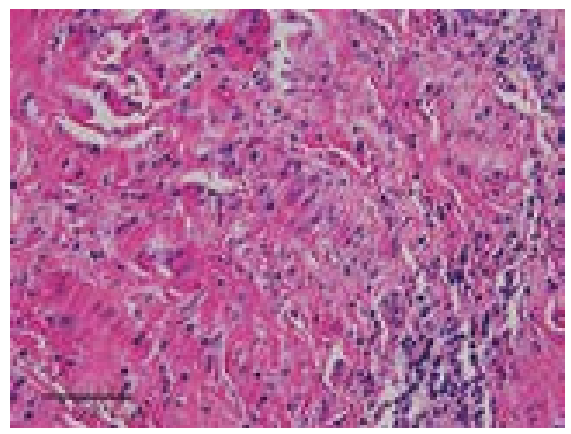

Figura 4 - Biopsia dural evidenciando paquimeningite linfo-histiocítica crônica sem infiltrado granulomatoso ou necrose ${ }^{12}$.

\section{REFERÊNCIAS}

1. Brodal P, 2010, The Central Nervous System, Oxford University Press.

2. Charcot JM, Joffroy A. Deux cas d'atrophie musculaire progressive avec lésions de la substance gris et des faisceaux antériolatéraux de la melle épinière. Arch Physiol Norm Pathol 1869;2:354-367, 629-649, 744-769.

3. Naffziger HC, Stern WE. Chronic pachymeningitis: report of a case and review of the literature. Arch Neurol Psychiatr 1949;62:383-411.

4. M.J. Kupersmith, V. Martin, G. Heller, A. Shah, H.J. Mitnick, Idiopathic hypertrophicpachymeningitis, Neurology 62 (2004) 686-694.

5. T. Yonekawa, H. Murai, S. Utsuki, et al., A nationwide survey of hypertrophicpachymeningitis in Japan, J. Neurol. Neurosurg. Psychiatry (2013).

6. Z.S. Wallace, M.N. Carruthers, A. Khosroshahi, et al., IgG4-related disease and hypertrophic pachymeningitis, Medicine (Baltimore) 92 (2013) 206-216.

7. S. Rossi, F. Giannini, A. Cerase, et al., Uncommon findings in idiopathic hypertrophiccranial pachymeningitis, J. Neurol. 251 (2004) 548-555.

8. M.J. Thurtell, A.B. Keed, M. Yan, T. Gottlieb, J.M. Spies, G.M. Halmagyi, Tuberculous cranial pachymeningitis, Neurology 68 (2007) 298-300.

9. Nayak, Lakshmi, Lauren E. Abrey, and Fabio M. Iwamoto. "Intracranial dural metastases." Cancer 115.9 (2009): 1947-1953.

10. Hahn, Lewis D., Robert Fulbright, and Joachim M. Baehring. "Hypertrophic pachymeningitis." Journal of the Neurological Sciences 367 (2016): 278-283.

11. Yonekawa, Tomomi, et al. "A nationwide survey of hypertrophic pachymeningitis in Japan." J NeurolNeurosurg Psychiatry (2013): jnnp-2013.

12. Wasilewski, A."Teaching Neurolmages: Idiopa- 
thic hypertrophic pachymeningitis." Neurology November 29, 2016 vol. 87 no. 22 e270-e271.

13. A. Rudnik, D. Larysz, J. Gamrot, A. Skorupa, G. Bierzynska-Macyszyn, P. Bazowski, Idiopathic hypertrophic pachymeningitis - case report and literature review, Folia Neuropathol. 45 (2007) 36-42.

14. Yoon, Byung-Nam, et al. "Neuro-Behçet's Disease Presenting as Hypertrophic Pachymeningitis." Experimental neurobiology 24.3 (2015): 252-255.

15. Hsu, Hui-Ting, et al. "Teaching Neuroimages: Idiopathic hypertrophic spinal pachymeningitis mimicking epidural lymphoma." Neurology 84.9 (2015): e67-8.

16. Lowden, Max R., and David Gill. "Teaching Neurolmage: Idiopathic hypertrophic spinal pachymeningitis." Neurology 72.5 (2009): e27-e27.

17. Lu, Lucy X., et al. "IgG4-related hypertrophic pachymeningitis: clinical features, diagnostic criteria, and treatment." JAMA neurology 71.6 (2014): 785-793.

18. Khosroshahi A, Carruthers MN, Deshpande V, Unizony S, Bloch DB, Stone JH. Rituximab for the treatment of IgG4-related disease: lessons from 10 consecutive patients. Medicine (Baltimore). 2012;91(1):57-6.

19. Aburahma SK, Anabtawi AG, Al Rimawi HS, Elheis MA, Mohtaseb AH. diopathic Hypertrophic Pachymeningitis in a Chlild With Hydrocephalus. Pediatr Neurol. 2009 Jun;40(6):457-60).

1- Serviço de Neurologia Clínica do HSI

Endereço para correspondência:

tiagossq@hotmail.com 